Artículo

\title{
Rendimiento y estabilidad de genotipos de soya para el trópico de México
}

Nicolás Maldonado Moreno

Juan Samuel Guadalupe Jesús Alcalá Rico ${ }^{\S}$

Guillermo Ascencio Luciano

Julio César García Rodríguez

Campo Experimental Las Huastecas-INIFAP. Carretera Tampico-Mante km 55, Villa Cuauhtémoc, Tamaulipas, México. CP. 89610. (maldonado.nicolas@inifap.gob.mx; ascencio.guillermo@inifap.gob.mx; garcia.juliocesar@inifap.gob.mx).

${ }^{\S}$ Autor para correspondencia: alcala.juan@inifap.gob.mx.

\section{Resumen}

Actualmente el cambio climático obliga a los fitomejoradores a desarrollar genotipos adaptados a mega-ambientes, lo que garantiza la correcta producción del cultivo. El objetivo de este estudio fue determinar el potencial en rendimiento de grano y estabilidad de genotipos de soya. Para estos fines, se evaluaron 15 genotipos de soya (siete variedades y ocho líneas experimentales) a través de cuatro años $(2014,2015,2016$ y 2017), en un diseño látice cuadrado 5x5 con tres repeticiones. El análisis de varianza reveló diferencias significativas en años, genotipos y en la interacción genotipos por años. Siendo la fuente de variación años, la que tuvo mayor impacto en el rendimiento con un $84.3 \%$, seguido de la interacción genotipos por años (10\%) y de genotipos (5.6\%). Asimismo, las condiciones del 2014 fueron más propicias para que los genotipos tuvieran un mayor rendimiento. Con relación a los genotipos, el G15, G7, G5 y G2 presentaron los mayores valores de rendimiento a través de los cuatro años. En cuanto a estabilidad y rendimiento el método de rendimiento relativo y la representación Biplot GGE coincidieron en que los genotipos que poseen estas dos características son G7 y G2. Por otro lado, se formaron dos mega-ambientes siendo el genotipo G15 el ganador respecto a su desempeño en el primer mega-ambiente donde estuvieron incluidos los años 2015, 2016 y 2017, de la misma forma el genotipo G5 fue el ganador en el segundo mega-ambiente constituido por el año 2014. Los dos métodos al complementarse explicaron mayormente la variación fenotípica del rendimiento.

Palabras clave: biplot, genotipo ideal, rendimiento relativo.

Recibido: julio de 2021

Aceptado: septiembre de 2021 


\section{Introducción}

La soya [Glycine $\max$ (L.) Merrill] es uno de los cultivos de mayor importancia en el mundo debido a su diversidad de usos y actualmente, la principal fuente de aceite comestible entre los cultivos de semillas oleaginosas (Bhartiya et al., 2014). Se puede adaptar a diversos climas, aunque se desarrolla de manera óptima en regiones tropicales (Pecina et al., 2005). Se cultiva principalmente en Norteamérica, Sudamérica y Asia, sin embargo los principales países exportadores son Estados Unidos y Brasil (Kumudini, 2010). De acuerdo a la demanda creciente del cultivo se busca genotipos superiores que posean potencial de rendimiento y estabilidad para lo cual se emplean ensayos en varios años y localidades (Lu'quez et al., 2002; Smith et al., 2005; Yang et al., 2009).

En este aspecto uno de los principales desafíos que enfrentan los fitomejoradores es la respuesta diferencial de los genotipos en función del ambiente (Kang y Gorman, 1989), conocido como interacción genotipo por ambiente (GEI). La expresión de cada característica en un cultivo es el resultado del efecto del genotipo (G), el efecto del ambiente (E) y la GEI (Yan y Tinker, 2005). Pero la evaluación genotípica se limita sólo a los efectos principales de G, mientras que la GEI se ignora sin considerar la estabilidad de los genotipos (Yan y Tinker, 2006; Bhartiya et al., 2017).

La GEI por medio del uso de las pruebas multiambiente (MET) permite evaluar con precisión el rendimiento de los cultivos en diferentes ambientes, predecir el nivel de rendimiento y examinar la estabilidad de los genotipos para la selección de mejores genotipos en los programas de mejoramiento (Magari y Kang, 1993; Ebdon y Gauch, 2002; Mustapha y Bakari, 2014). Se han desarrollado técnicas estadísticas para estudiar la GEI, entre ellas están los modelos univariados, como la pendiente de regresión, la desviación de la regresión, la varianza ambiental y la estabilidad del rendimiento, y modelos multivariados, como el efecto principal del genotipo más genotipo por ambiente (GGE) y efectos principales aditivos e interacción multiplicativa (AMMI) (Finlay y Wilkinson, 1963; Eberhart y Russell, 1966; Kang, 1993; Yan, 2001).

Estos dos últimos modelos se basan en el análisis de componentes principales (PC) y tienen la capacidad de clasificar los genotipos en base a alguna característica de interés y a la estabilidad que presenta esta esta característica en cada genotipo al evaluarlos en diferentes ambientes (Casanoves et al., 2005). Para fines de investigación y para delinear mega-ambientes, el modelo AMMI, el modelo SREG y SHMM se consideran igualmente efectivos (Gauch et al., 2008). La presente investigación se realizó con el objetivo de comparar el método de rendimiento relativo y la representación Biplot GGE e identificar genotipos de soya con buen rendimiento y estabilidad bajo condiciones de temporal en la región sur de Tamaulipas, México.

\section{Materiales y métodos}

\section{Ubicación del sitio experimental}

La investigación se desarrolló en los ciclos primavera-verano (P-V) del año 2014 al 2017, bajo condiciones de temporal en el Instituto Nacional de Investigaciones Forestales, Agrícolas y Pecuarias (INIFAP), Campo Experimental Las Huastecas, ubicado a $22^{\circ} 33^{\prime} 57.88^{\prime \prime}$ latitud norte y $98^{\circ} 09^{\prime} 52.47^{\prime \prime}$ ' longitud oeste con una altitud de $17 \mathrm{msnm}$. Esta región presenta clima trópico húmedo semicálido, con una temperatura media anual de $24.5^{\circ} \mathrm{C}$ y una precipitación de $842 \mathrm{~mm}$. 


\section{Material vegetal}

Se utilizaron 15 genotipos entre los cuales se incluyeron variedades y líneas experiméntales del área de mejoramiento genético de soya del Programa de Oleaginosas Anuales del INIFAP, las cuales presentan diferentes características de interés agronómico como lo es sanidad, porte, producción de grano y adaptabilidad a la región Huasteca (Cuadro 1).

Cuadro 1. Identificación de las variedades y líneas experimentales.

\begin{tabular}{cc}
\hline Id & Genotipos \\
\hline G1 & H02-1337 \\
G2 & H02-1987 \\
G3 & H02-2082 \\
G4 & H10-0556 \\
G5 & H10-3056 \\
G6 & H98-1240 \\
G7 & H98-1325 \\
G8 & H98-1521 \\
G9 & Huasteca 100 \\
G10 & Huasteca 200 \\
G11 & Huasteca 300 \\
G12 & Huasteca 400 \\
G13 & Huasteca 600 \\
G14 & Huasteca 700 \\
G15 & Tamesí \\
\hline
\end{tabular}

\section{Metodología}

Se realizó la preparación del terreno iniciando con el barbecho a una profundidad de $30 \mathrm{~cm}$, seguido de dos pasos de rastra a los 20 días después del barbecho y finalmente se ejecutó el surcado a 76 cm de separación.

La siembra se efectuó en parcelas de 4 surcos de $5 \mathrm{~m}$ de longitud con una densidad de 250000 plantas ha ${ }^{-1}$. El resto del manejo agronómico se realizó de acuerdo con el paquete tecnológico de soya para el sur de Tamaulipas (Maldonado, 2017). Al momento de la cosecha se registró el rendimiento de grano con un contenido de humedad de $14 \%$ en unidades de $\mathrm{kg} \mathrm{ha}^{-1}$.

\section{Diseño}

El experimento se estableció bajo un diseño látice cuadrado 5x5, con tres repeticiones. Se consideraron dos factores, el primero fueron los genotipos quienes se aleatorizaron en el látice y el segundo los años. 


\section{Análisis estadísticos}

Con la información de cada ambiente se realizó el análisis de varianza combinado, y se aplicó la prueba de Tukey con 0.05 de nivel de significancia para la comparación de las medias de rendimiento de los genotipos y años. Para estimar la estabilidad del rendimiento se utilizó el método de rendimiento relativo (RR), este método consiste en expresar el rendimiento de cada genotipo en cada ambiente en forma relativa al promedio del ambiente determinado, asignándole a este último el valor de 100. Los materiales genéticos que posean menor rendimiento que el promedio de todos los genotipos en el mismo ambiente tendrán valores de RR menores a 100, mientras que los que tengan mayor rendimiento, tendrán valores mayores a 100.

La desviación estándar, calculada como la raíz cuadrada de la varianza de los rendimientos relativos de cada material genético a través de los ambientes, es la medida de la estabilidad. Los genotipos más estables serán los que tengan menor desviación estándar (Yau y Hamblin, 1994). Se elaboró un gráfico de líneas para la interpretación de datos climáticos. Para la interacción genotipo por ambiente se realizaron análisis de componentes principales a partir de los cuales se desarrollaron gráficos Biplot GGE (genotipo más genotipo por ambiente) (Yan et al., 2007). Para los análisis se usó el paquete estadístico R versión 3.6 (R, 2020) y SAS versión 9.4 (SAS, 2014).

\section{Resultados y discusión}

\section{Análisis de varianza}

En el presente trabajo se observó que las repeticiones dentro de los años no influyeron en el rendimiento por lo que se puede asumir que las condiciones de terreno fueron homogéneas. Por otro lado, los años, así como los genotipos y su interacción además de los dos primeros componentes principales mostraron diferencias significativas $(p \leq 0.01)$, esto podría deberse a la diversidad genética que presentan los genotipos, las condiciones particulares que presentaron los años y la respuesta diferente de los genotipos en cada año.

En lo que respecta a la heredabilidad, este carácter presentó valores bajos donde el componente ambiental contribuyó con la mayor proporción de la variación total, por lo que se asume que el carácter está controlado por varios genes de efecto pequeño (Cuadro 2). Asimismo, la contribución relativa de la varianza del componente genotipos por años fue mayor en comparación con el componente de varianza genotipos. Estos resultados son similares a los reportado por Shukla et al. (2015); Vaezi et al. (2017) quienes mencionan que la mayor parte de la variación es atribuible a los efectos ambientales seguidos de la interacción genotipo por ambiente y genotipo.

Cuadro 2. Componentes principales y cuadrados medios del análisis de varianza para la variable rendimiento.

\begin{tabular}{ccccc}
\hline FV & GL & CM & SC $(\%)$ \\
\hline Años & 3 & 22496734 & ns & 74.7 \\
Rep(Años) & 8 & 99660 & $* *$ & 0.9 \\
Gen & 14 & 322278 & $* *$ & 5 \\
Gen*Años & 42 & 191069 & 8.9 \\
\hline
\end{tabular}




\begin{tabular}{ccccc}
\hline FV & GL & CM & & SC (\%) \\
\hline CP1 & 16 & 248131 & $* *$ & \\
CP2 & 14 & 202728.5 & ns & \\
CP3 & 12 & 96615.4 & & 10.5 \\
Error & 112 & 84894 & \\
$\mathrm{H}^{2}(\%)$ & & 8 & \\
$\mathrm{R}^{2}(\%)$ & & 89 & \\
$\mathrm{CV}(\%)$ & & 11.2 & \\
\hline
\end{tabular}

${ }^{*}=$ significativo al $0.05 ;{ }^{* *}=$ significativo al $0.01 ; \mathrm{ns}=$ no significativo; $\mathrm{FV}=$ fuente de variación $; \mathrm{GL}=$ grados de libertad; $\mathrm{Rep}=$ repetición; $\mathrm{Gen}=$ genotipos; $\mathrm{CP}=$ componentes principales; $\mathrm{R}^{2}=$ coeficiente de determinación; $\mathrm{CV}=$ coeficiente de variación; $\mathrm{CM}=$ cuadrados medios; $\mathrm{SC}(\%)=$ porcentaje de suma de cuadrados.

\section{Comparación de medias y estabilidad}

De acuerdo con el Cuadro 3, se observó una variación en los promedios generales de rendimiento. En cuanto a los años en promedio 2014 presentó el valor más alto de rendimiento siendo superior que el promedio del resto de los años en 32.6\%. Asimismo, el menor valor lo obtuvo el 2016, esto estuvo asociado a la precipitación pluvial ocurrida durante el ciclo del cultivo, y en especial la cantidad y distribución de las lluvias ocurridas durante el periodo de llenado de grano. Esto coincide con López-Castañeda (2011), quien menciona que el estrés hídrico reduce la conductancia estomática y la tasa de asimilación neta durante el llenado de grano, lo cual reduce el rendimiento.

Cuadro 3. Rendimiento relativo de genotipos de soya durante cuatro años.

\begin{tabular}{|c|c|c|c|c|c|c|c|c|c|c|c|}
\hline \multirow{2}{*}{ Gen } & \multicolumn{2}{|c|}{2014} & \multicolumn{2}{|c|}{2015} & \multicolumn{2}{|c|}{2016} & \multicolumn{2}{|c|}{2017} & \multirow{2}{*}{$\begin{array}{l}\text { Rend } \\
\text { promedio }\end{array}$} & \multirow{2}{*}{$\mathrm{RR}$} & \multirow{2}{*}{$\mathrm{s}^{2}$} \\
\hline & Rend & $\mathrm{R}(\%)$ & Rend & $\mathrm{R}(\%)$ & Rend & $\mathrm{R}(\%)$ & Rend & $\mathrm{R}(\%)$ & & & \\
\hline G15 & 3613.8 & 106 & 3118 & 134 & 1878.3 & 108 & 3458.2 & 123 & $3017.1 \mathrm{a}$ & 118 & 13 \\
\hline G7 & 3500.4 & 103 & 2785.4 & 119 & 1784.7 & 103 & 3000.8 & 107 & $2767.8 \mathrm{ab}$ & 108 & 7.8 \\
\hline G5 & 3908.7 & 115 & 2237.2 & 96 & 1903.5 & 110 & 2842 & 101 & $2722.9 \mathrm{abc}$ & 106 & 8.5 \\
\hline $\mathrm{G} 2$ & 3464.6 & 102 & 2358.8 & 101 & 1705.6 & 98 & 2962.1 & 106 & $2622.8 \mathrm{abc}$ & 102 & 2.9 \\
\hline G14 & 3518.8 & 104 & 2322.1 & 100 & 1950.2 & 113 & 2617 & 93 & $2602 \mathrm{bc}$ & 102 & 8.1 \\
\hline G9 & 3798.6 & 112 & 2011.1 & 86 & 1670 & 96 & 2833.2 & 101 & $2578.2 \mathrm{bc}$ & 99 & 10.6 \\
\hline $\mathrm{G} 3$ & 3636.1 & 107 & 2337.1 & 100 & 1559.6 & 90 & 2752.7 & 98 & $2571.4 \mathrm{bc}$ & 99 & 7 \\
\hline $\mathrm{G} 1$ & 3095.1 & 91 & 2514.9 & 108 & 1742.5 & 101 & 2906.8 & 104 & $2564.8 \mathrm{bc}$ & 101 & 7.1 \\
\hline G13 & 3124.6 & 92 & 2450.5 & 105 & 1723.6 & 99 & 2905.3 & 103 & $2551 \mathrm{bc}$ & 100 & 5.9 \\
\hline G10 & 2903.1 & 85 & 1912.9 & 82 & 2281 & 132 & 2956.2 & 105 & $2513.3 \mathrm{bc}$ & 101 & 22.8 \\
\hline G6 & 3179.3 & 94 & 2270.9 & 97 & 1567.8 & 90 & 2801.7 & 100 & $2454.9 \mathrm{bc}$ & 95 & 4.1 \\
\hline G8 & 3525.3 & 104 & 2349.8 & 101 & 1530.5 & 88 & 2368.7 & 84 & $2443.6 \mathrm{bc}$ & 94 & 9.4 \\
\hline G11 & 3010.5 & 89 & 2385.7 & 102 & 1753 & 101 & 2412.3 & 86 & $2390.4 \mathrm{bc}$ & 94 & 8.4 \\
\hline $\mathrm{G} 4$ & 3419.9 & 101 & 1835.2 & 79 & 1530.3 & 88 & 2706.4 & 96 & $2373 \mathrm{bc}$ & 91 & 9.7 \\
\hline G12 & 3280 & 97 & 2080.7 & 89 & 1419.8 & 82 & 2585.9 & 92 & $2341.6 \mathrm{c}$ & 90 & 6.1 \\
\hline$\overline{\mathrm{x}}$ & $3398.6 \mathrm{a}$ & 100 & $2331.4 \mathrm{c}$ & 100 & $1733.4 \mathrm{~d}$ & 100 & $2807.3 \mathrm{~b}$ & 100 & & & \\
\hline
\end{tabular}

Genotipos de soya con la misma letra son estadísticamente iguales; Gen: genotipos; Rend= rendimiento; $\mathrm{R}(\%)=$ porcentaje relativo; $\overline{\mathrm{x}}=$ promedio; $\mathrm{RR}=$ rendimiento relativo; $\mathrm{s}^{2}=$ desviación estándar del rendimiento relativo. 
Por otro lado, la temperatura no fue un factor limitante dado que descendió considerablemente al llegar a la etapa de madurez fisiológica (R8) (Figura 1). Los genotipos que sobresalieron en cuanto a su rendimiento fueron G15 (Tamesí), G7 (H98-1325), G5 (H10-3056) y G2 (H02-1987) superando al promedio del resto de genotipos en 17.5, 10, 8.6 y $5.1 \%$ respectivamente. Estos porcentajes en cuanto a años y genotipos confirma el impacto que tiene el ambiente en la expresión del carácter. En cuanto al método RR los genotipos que presentaron mayor rendimiento relativo y estabilidad fueron G7 (H98-1325), G5 (H10-3056) y G2 (H02-1987) presentando valores superiores a 100 en RR y una menor desviación estándar en comparación con el promedio.

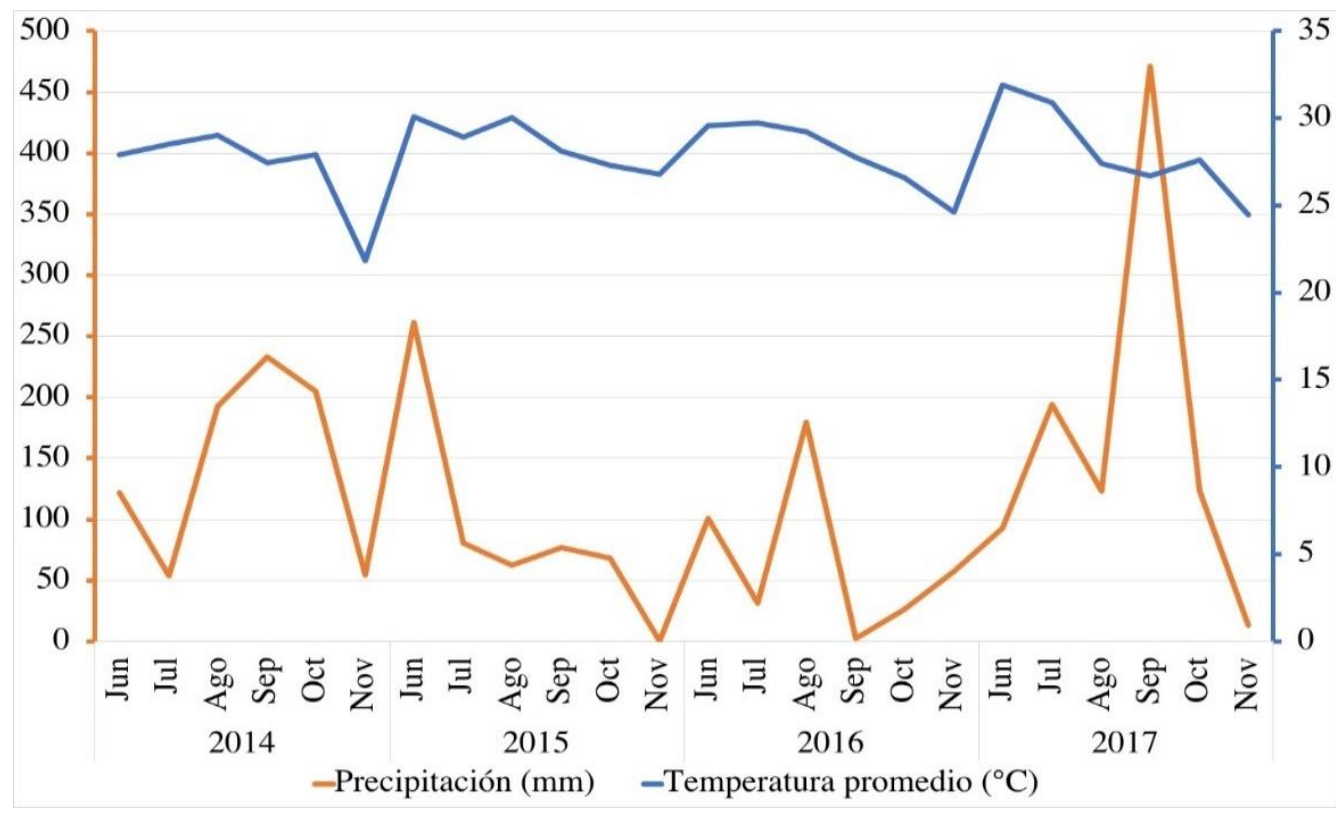

Figura 1. Datos climáticos del ciclo P-V del año 2014 al 2017 en el INIFAP-Las Huastecas.

En lo que respecta los componentes principales (CP) para el rendimiento, los dos primeros $\mathrm{CP}$ explicaron $75.15 \%$ de la variación total. El CP1 explicó la mayor variación (46.94\%) seguido del CP2 (28.21\%). Para representar la estabilidad en este biplot, el eje de la abscisa de la coordenada del medio ambiente (AEC) es la línea de una sola flecha horizontal verde que pasa por el origen del biplot. Con respecto al eje de la ordenada AEC está representado con la línea vertical verde que pasa a través del origen biplot y es perpendicular a la abscisa AEC, de esta manera los genotipos que presentan mayor distancia sobre la abscisa AEC en cualquier dirección serán más inestables (Yan et al., 2007).

Por lo tanto, el genotipo G2 (H02-1987), seguido del G14 (Huasteca 700) y G7 (H98-1325) fueron los más estables, mientras que los genotipos G10 (Huasteca 200), G9 (Huasteca 100) y G5 (H103056) correspondieron a ser altamente inestables en todos los años para la variable rendimiento (Figura 2). En este sentido, Brar et al. (2010) menciona que el genotipo ideal debe presentar una alta estabilidad para asegurar una adaptabilidad a una región objetivo y minimizar el riesgo de pérdida de rendimiento debido a las condiciones ambientales. 


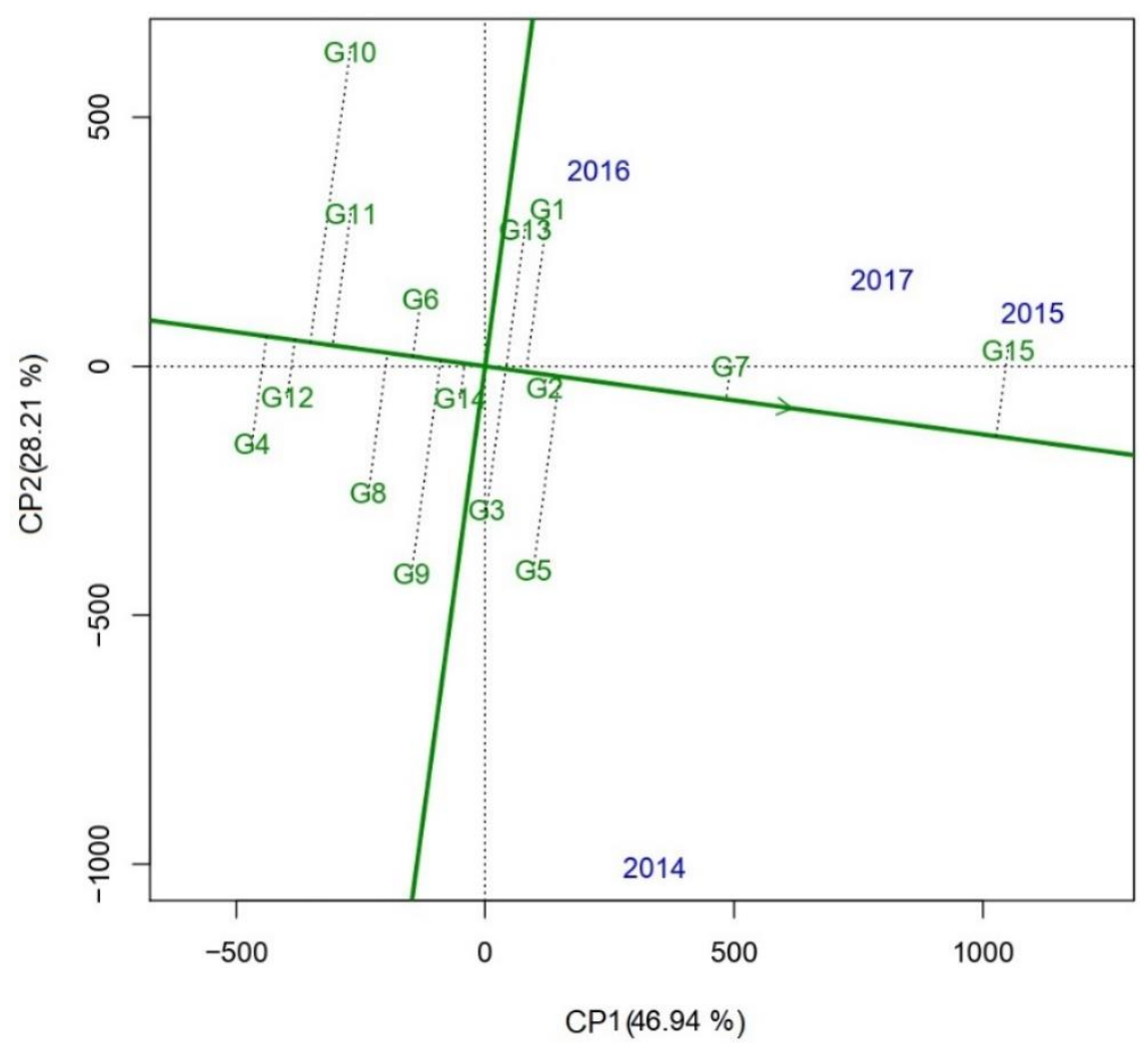

Figura 2. Estabilidad de genotipos a través de los años.

\section{Genotipo ideal}

En este aspecto, los cuatro genotipos que más se acercaron a lo ideal fueron el G15 (Tamesí), teniendo el rendimiento promedio más alto, seguido del G7 (H98-1325), G5 (H10-3056) y G2 (H02-1987). Por otra parte, los genotipos G3 (H02-2082), G13 (Huasteca 600) y G14 (Huasteca 700) tuvieron un comportamiento similar al promedio general. Sin embargo, G4 (H10-0556), G12 (Huasteca 400) y G11 (Huasteca 300) se consideraron como los menos ideales ya que presentaron en promedio el menor rendimiento a través los años.

El genotipo G10 también se encuentra en esta última lista por haber presentado los valores más extremos (altos y bajos) a lo largo de los años (Figura 3). Estas observaciones se relacionan con Farshadfar et al. (2013), quienes mencionan que un genotipo ideal es aquel que tiene un alto rendimiento medio de grano y una alta estabilidad. Cabe resaltar, que los genotipos se distribuyeron en los cuatro cuadrantes, lo que indica la diversidad genética que estos poseen, la cual es indispensable en un programa de mejoramiento genético (Figura 3). Rimieri (2017), menciona que la variabilidad disponible para seleccionar se encuentra en genotipos previamente adaptados, ya que la variabilidad de las especies en estado silvestre generalmente no puede utilizarse directamente. 


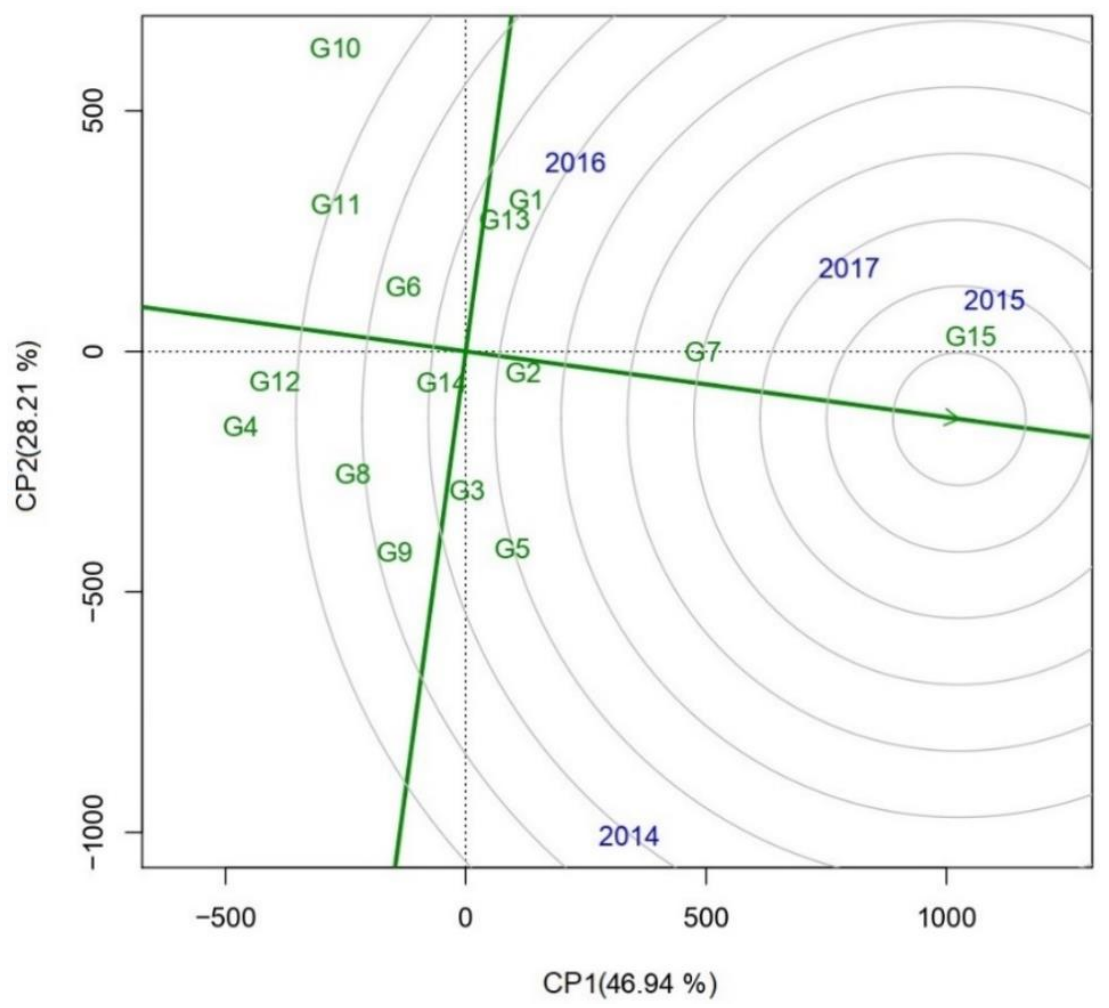

Figura 3. Biplot GGE del genotipo ideal de soya.

\section{Mega-ambientes}

La relación que presentaron los años dependió del coseno de ángulo de los vectores el cual se aproxima al coeficiente de correlación, donde el ángulo agudo indicó una relación positiva, sin embargo el ángulo obtuso reveló una asociación negativa y los ángulos rectos significó que no existió ninguna (Yan, 2002; Haider et al., 2017), pudiéndose formar dos grupos de megaambientes: el primer grupo estuvo conformado por los años 2015, 2016 y 2017, asimismo el segundo grupo se conformó por el año 2014. Este último presentó un ángulo de recto a obtuso con respecto a los años del primer grupo. Por otro lado, los años 2014 y 2015 fueron los más discriminatorios debido a la longitud de sus vectores, los cuales fueron mayores al resto de años. Este dato es indispensable para reducir costos y mejorar la precisión de la selección (Imtiaz et al., 2013).

Para poder observar los mejores genotipos en cada ambiente y grupos de ambientes se utilizó el patrón 'qué ganó dónde'. El biplot se dividió en cinco sectores, donde los cuatro años cayeron en dos, indicando la presencia de interacción cruzada significativa. De acuerdo con Yan et al. (2007), cuando diferentes ambientes caen en distintos sectores, implica que existen genotipos de alto rendimiento para esos sectores. Asimismo, Yan y Kang (2003) mencionan que a partir de la vista poligonal se puede observar de mejor manera la presencia o ausencia de interacción cruzada genotipo por ambiente que explica la existencia de distintos mega-ambientes. 
Por otro lado, el genotipo G15 (Tamesí) dio un alto rendimiento en los años 2015, 2016 y 2017, mientras que el genotipo G5 (H10-3056) proyectó el mejor rendimiento en el año 2014, lo que sugiere que se pueden desarrollar genotipos especializados para ambientes específicos (Figura 4). Este comportamiento es debido a que los genotipos ubicados en los vértices de cada sector indicaron un mejor o peor comportamiento en uno u otro ambiente (Yan et al., 2000; Dia et al., 2016).

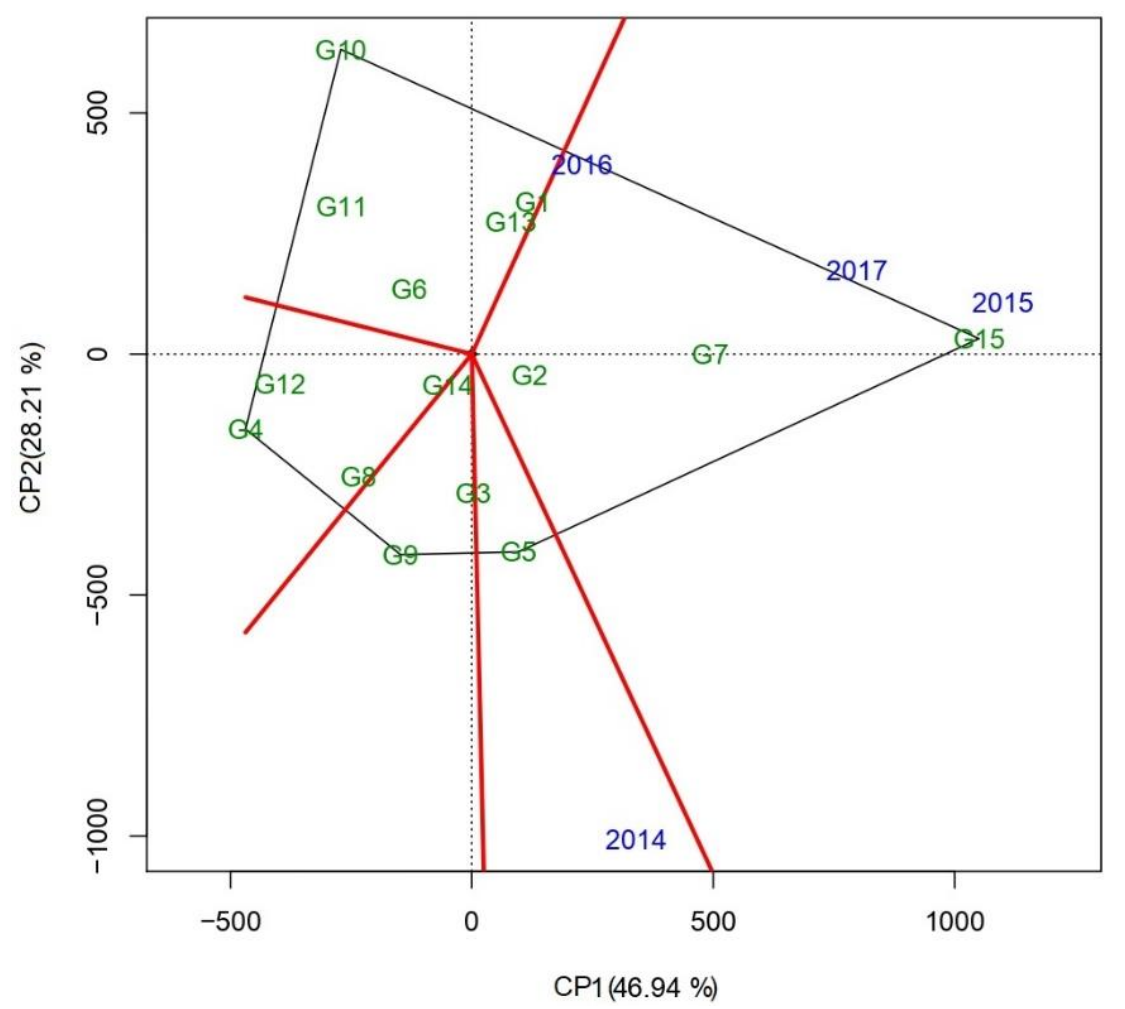

Figura 4. Biplot 'qué (que genotipo)-ganó-dónde’ de 14 genotipos en cuatro años.

De acuerdo con los resultados anteriores los genotipos se pudieron clasificar en cuatro grupos. El primer grupo estuvo conformado por los genotipos G15 (Tamesí), G7 (H98-1325) y G2 (H021987) siendo estos los más rendidores y estables que son las principales características para el mejoramiento. El segundo se integraron los genotipos G1 (H02-1337), G13 (Huasteca 600) y G5 (H10-3056) que fueron rendidores pero inestables, ideales para la realización de cruzamientos y selección.

El tercer grupo estuvo constituido por los genotipos G12 (Huasteca 400), G4 (H10-0556), G6 (H981240) y G14 (Huasteca 700) estables, pero con rendimiento igual o menor al promedio. Por último, el cuarto grupo, se formó por los genotipos G11 (Huasteca 300), G9 (Huasteca 100), G8 (H981521), G3 (H02-2082) y G10 (Huasteca 200) inestables con rendimiento igual o menor al promedio, estos dos últimos grupos serían descartados para un programa de mejoramiento genético. Con relación a esto, López et al. (2011) menciona que los estudios de adaptabilidad y estabilidad del rendimiento son de vital importancia para determinar la respuesta de los genotipos en diferentes localidades, años y ciclos del cultivo. 


\section{Conclusiones}

El método RR y la representación Biplot GGE resultaron ser eficientes al complementarse, ya que por un lado la primera toma en cuenta los genotipos y parte del ambiente; sin embargo, en el segundo se determina el efecto de genotipo más su interacción con el ambiente. Los genotipos G15, G7 y G2 contribuirán con mayor potencial de rendimiento por unidad de superficie y buena estabilidad para el trópico de México.

\section{Literatura citada}

Bhartiya, A.; Aditya, J. P.; Kumari, V.; Kishore, N.; Purwar, J. P.; Agrawal, A. and Kant, L. 2017. GGE biplot \& AMMI analysis of yield stability in multi-environment trial of soybean [Glycine $\max$ (L.) Merrill] genotypes under rainfed condition of north western Himalayan hills. J. Anim. Plant Sci. 27(1):227-238.

Bhartiya, A.; Singh, K.; Aditya, J. P.; Puspendra and Gupta, M. 2014. Residual relative heterosis and heterobeltiosis for different agro morphological traits as selection index in early segregating generation of soybean [Glycine max (L.) Merrill] crosses. Soybean res. 12(1):28-35.

Brar, K. S.; Singh, P.; Mittal, V. P.; Singh, P.; Jakhar, M. L.; Yadav, Y.; Sharma, M. M.; Shekhawat, U. S. and Kumar, C. 2010. GGE biplot analysis for visualization of mean performance and stability for seed yield in taramira at diverse locations in India. J. Oilseed Brassica. 1(2):66-74.

Casanoves, F.; Baldessari, J. and Balzarini, M. 2005. Evaluation of multienvironment trials of peanut cultivars. Crop Sci. 45(1):18-26. doi:10.2135/cropsci2005.0018.

Dia, M.; Wehner, T. C.; Hassell, R.; Price, D. S.; Boyhan, G. E.; Olson, S.; King, S.; Davis, A. R. and Tolla, G. E. 2016. Genotype environment interaction and stability analysis for watermelon fruit yield in the United States. Crop Sci. 56(4):1645-1661. doi:10.2135/ cropsci2015.10.0625.

Ebdon, J. S. and Gauch, H. G. 2002. Additive main effect and multiplicative interaction analysis of national turfgrass performance trials. Crop Sci. 42(2):497-506. doi:10.2135/cropsci200 2.0489 .

Eberhart, S. T. and Russell, W. A. 1966. Stability parameters for comparing varieties. Crop Sci. 6(1):36-40. doi: 10.2135/cropsci196 6.0011183X000600010011x.

Farshadfar, E.; Rashidi, M.; Jowkar, M. M. and Zali, H. 2013. GGE Biplot analysis of genotype environment interaction in chickpea genotypes. Eur. J. Exp. Biol. 3(1):417-423.

Finlay, K. W. and Wilkinson, G. N. 1963. The analysis of adaptation in a plant breeding programme. Aust. J. Agric. Res. 14(6):742-754. doi: 10.1071/AR9630742.

Gauch, H. G.; Piepho, H. P. and Annicchiarico, P. 2008. Statistical analysis of yield trials by AMMI and GGE: Further considerations. Crop Sci. 48(3):866-889. doi:10.2135/cropsci2007. 09.0513.

Haider, Z.; Akhter, M.; Mahmood, A. and Khan, R. A. R. 2017. Comparison of GGE biplot and AMMI analysis of multi-environment trial (MET) data to assess adaptability and stability of rice genotypes. African J. Agric. Res. 12(51):3542-3548. doi:10.5897/AJAR2017.12528.

Imtiaz, M.; Malhotra, R. S.; Singh, M. and Arslan, S. 2013. Identifying high yielding, stable chickpea genotypes for spring sowing: specific adaptation to locations and sowing seasons in the Mediterranean region. Crop Sci. 53(4):1472-1480. doi:10.2135/cropsci2012.10.0589. 
Kang, M. S. 1993. Simultaneous selection for yield and stability in crop performance trials: Consequences for growers. Agron. J. 85(3):754-757.

Kang, M. S. and Gorman, D. P. 1989. Genotype $\times$ environment interaction in maize. Agron. J. 81(4):662-664. doi: 10.2134/agron j1989.00021962008100040020x.

Kumudini, S. 2010. Soybean growth and development. The soybean: botany, production and uses. (Ed.) by Guriq Singh. Ludhiana, India. 23 p.

López-Castañeda, C. 2011. Variación en rendimiento de grano, biomasa y número de granos en cebada bajo tres condiciones de humedad del suelo. Trop. Subtrop. Agroecosyst. 14(3):907-918.

López, S. E.; Acosta, G. J. A.; Tosquy, V. O. H.; Salinas, P. R. A.; Sánchez, G. B. M.; Rosales, S. R.; González, R. C.; Moreno, G. T.; Villar, S. B. Cortinas; E. H. M. y Zandate, H. R. 2011. Estabilidad de rendimiento en genotipos mesoamericanos de frijol de grano en México. Rev. Mex. Cienc. Agríc. 2(1):29-40.

Lu'quez, J. E.; Aguirreza'bal, L. A. N.; Aguero, M. E. and Pereyra, V. R. 2002. Stability and adaptability of cultivars in non- balanced yield trials: comparison of methods for selecting 'high oleic' sunflower hybrids for grain yield and quality. J. Agron. Crop Sci. 188(4):225234. doi:10.1046/j.1439-037X.2002.00562.x.

Magari, R. and Kang, M. S. 1993. Genotype selection via a new yield stability statistic in maize yield trials. Euphytica. 70(1-2):105-111. doi: 10.1007/BF00029647.

Maldonado, M. N. 2017. Soya de temporal y riego para el sur de Tamaulipas ciclo P-V. Agenda Técnica Agrícola Tamaulipas. Instituto Nacional de Investigaciones Forestales, Agrícolas y Pecuarias (INIFAP). Ciudad de México. 92-105 pp.

Mustapha, M. and Bakari, H. R. 2014. Statistical evaluation of genotype by environment interactions for grain yield in millet (Penniisetum glaucum (L.) R. Br.). Int. J. Eng. Sci. 3(9):7-16.

Pecina, Q. V.; Maldonado, H. L.; Maldonado, M. N.; Simpson, J.; Martínez, V. O. y Gil, V. K. C. 2005. Diversidad genética en soya del Trópico Húmedo de México determinada con marcadores AFLP. Rev. Fitotec. Mex. 28(1):63-69.

R. 2020. R: A Language and environment for statistical computing. R foundation for statistical computing. Vienna, Austria. Retrieved from http://www.r-project.org/.

Rimieri, P. 2017. La diversidad y la variabilidad genéticas: dos conceptos diferentes asociados al germoplasma y al mejoramiento genético vegetal. BAG. J. Basic Appl. Gen. 28(2):7-13.

SAS. 2014. SAS: Business analytics and business intelligence software. NC, USA: SAS Inst. Retrieved from http://www.sas.com/en_us/home.html.

Shukla, S.; Mishra, B. K.; Mishra, R.; Siddiqui, A.; Pandey, R. and Rastogi, A. 2015. Comparative study for stability and adaptability through different models in developed high thebaine lines of opium poppy (Papaver somniferum L.). Ind. Crops prod. 74:875-886. doi:10.1016/j.indcrop.2015.05.076.

Smith, A. B.; Cullis, B. R. and Thompson, R. 2005. The analysis of crop cultivar breeding and evaluation trials: An overview of current mixed model approaches. J. Agric. Sci. 143(06):449-462. doi: 10.1017/S0021859605005587.

Vaezi, B.; Pour-Aboughadareh, A.; Mohammadi, R.; Armion, M.; Mehraban, A.; Hossein-Pour, T. and Dorii, M. 2017. GGE biplot and AMMI analysis of barley yield performance in Iran. Cereal res. Commun. 45(3):500-511. doi:10.1556/0806.45.2017.019.

Yan, W. 2001. GGE biplot a Windows application for graphical analysis of multienvironment trial data and other types of two-way data. Agron. J. 93(5):1111-1118. 
Yan, W. and Tinker, N. A. 2005. An integrated biplot analysis system for displaying, interpreting and exploring genotype environment interaction. Crop Sci. 45(3):1004-1016. doi:10.2135/cropsci2004.0076.

Yan, W. and Kang, M. S. 2003. GGE biplot analysis: a graphical tool for breeders, geneticists, and agronomists. CRC Press. Boca Raton, London, New York, Washington, D.C. retrieved from https://content.taylorfrancis.com/books/download?dac=c2009-0-09571-8\&isbn=978 0429122729\&format=googlepreviewpdf. 263 p.

Yan, W. 2002. Singular-value partitioning in biplot analysis of multienvironment trial data. Agron. J. 94(5):990-996. doi:10.2134/AGRONJ2002.9900.

Yan, W.; Hunt, L. A.; Sheng, Q. and Szlavnics, Z. 2000. Cultivar evaluation and mega-environment investigation based on the GGE biplot. Crop Sci. 40(3):597-605. doi:10.2135/cropsci2000. 403597x.

Yan, W.; Kang, M. S.; Ma, B.; Woods, S. and Cornelius, P. L. 2007. GGE biplot vs. AMMI analysis of genotype-by-environment data. Crop sci. 47(2):643-655. doi:10.2135/cropsci2006. 06.0374.

Yan, W. and Tinker, N. A. 2006. Biplot analysis of multi-environment trial data: principles and applications. Can. J. Plant Sci. 86(3):623-645. doi:10.4141/P05-169.

Yang, R. C.; Crossa, J.; Cornelius, P. L. and Burgueño, J. 2009. Biplot analysis of genotype environment interaction: proceed with caution. Crop Sci. 49(5):1564-1576. doi:10.2135/cropsci2008.11.0665.

Yau, S. K. and Hamblin, J. 1994. Relative yield as a measure of entry performance in variable environments. Crop Sci. 34(3):813-817. doi:10.2135/cropsci1994.0011183X0034000 $30038 x$. 